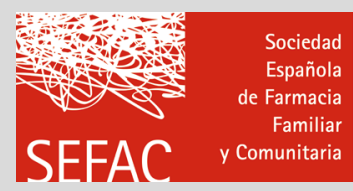

\title{
Convierte tu sueño profesional en realidad en el octavo congreso de SEFAC en Alicante
}

\author{
Maite Climent Catalá ${ }^{1,2}$, Rosa Prats Mas ${ }^{3}$ \\ 1. Presidenta del comité organizador del VIII Congreso Nacional de Farmacéuticos Comunitarios. 2. Presidenta de SEFAC Comunitat Valenciana. \\ 3. Presidenta del comité científico del VIII Congreso Nacional de Farmacéuticos Comunitarios.
}

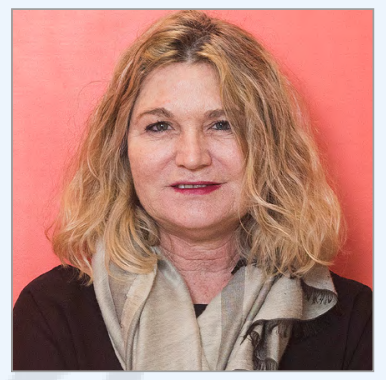

Maite Climent

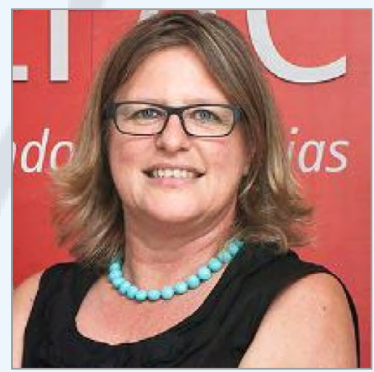

Rosa Prats

\section{PALABRAS CLAVE}

Farmacéutico comunitario, servicios profesionales

farmacéuticos, investigación, congreso

\section{KEYWORDS}

Community pharmacist, professional pharmaceutical services, research, conference
"Haz de tu vida un sueño y de tu sueño una realidad". Esta perla de sabiduría del autor de El principito, Antonie de Saint-Exupéry, refleja como pocas el genio de todos aquellos pioneros que fueron capaces de transformar su presente anticipándose al futuro. Francisco Martínez, fundador y primer presidente de SEFAC, se empeñó en hacer realidad su sueño, nuestro sueño, hace casi ya 20 años. Preocupado y sorprendido al constatar que en ningún congreso farmacéutico se presentaban comunicaciones firmadas por farmacéuticos comunitarios, contactó con colegas de todas partes de la geografía española que compartían su visión para levantar SEFAC, la única sociedad de ámbito nacional creada por y para el farmacéutico comunitario.

El ideal de farmacia asistencial al que dieron forma ese irrepetible grupo de soñadores es hoy una realidad, que aún necesita de estímulos para consolidarse y consolidar su sostenibilidad. Con esa finalidad nacieron, allá por el año 2004 en Tarragona, los congresos de SEFAC, foros de intercambio de conocimientos en los que perfilar los servicios asistenciales que demandaban los pacientes, difundir el potencial científico de los farmacéuticos comunitarios y reivindicar nuestro papel como agentes sanitarios de primer orden. Aunque la profesión ha virado con éxito en los tres últimos lustros su foco desde el medicamento al paciente, aún quedan retos pendientes, que acometemos con orgullo en la VIII edición del Congreso Nacional de Farmacéuticos Comunitarios, que se celebrará en el Auditorio de la Diputación de Alicante del 24 al 26 de mayo.

Como marca la tradición en los congresos de SEFAC, la cita alicantina supondrá un crecimiento de público -esperamos una afluencia que rondará los 1.500 congresistas- y de contenidos frente a ediciones anteriores. Del 24 al 26 de mayo se sucederán más de 60 sesiones (frente a las 50 de la pasada edición del Congreso en Zaragoza), divididas en 9 mesas redondas, 16 talleres formativos, 11 simposios, 14 sesiones prácticas, presentación de proyectos de la Sociedad como Conóceme (sobre el grado de conocimiento de los medicamentos por los adolescentes) o Alivia (dolor), entre otros y de iniciativas clave en materia sanitaria como Know your pulse, MMM o Predire... En definitiva, creemos que estamos ante el mejor escaparate de contenidos con evidencia científica de la farmacia comunitaria. También se ha incrementado sustancialmente la zona de exposición. Más de 60 entidades que creen y comparten nuestra visión de la farmacia asistencial nos acompañarán en Alicante, donde también se exhibirá una gran relación de pósteres científicos, de entre las más de 300 comunicaciones recibidas.

No queremos que nadie se sienta excluido de este sueño de una farmacia de servicios. No importa cuál sea tu condición: ya seas socio de SEFAC o no asociado, estudiante o profesional que ejerce fuera de España, tenemos una cuota de inscripción a tu medida que puedes consultar en la web del congreso, incluso si no se es farmacéutico; así como una variada oferta de contenidos científicos orientada a resolver tus inquietudes profesionales de presente y futuro. En el congreso se abordarán asuntos tan capitales, por relevancia y actualidad, como la atención farmacéutica domiciliaria, a la que dedicaremos el debate profesional del evento. En dicho debate, en el que intercambiaremos ideas y escucharemos las aportaciones de agentes sanitarios, Administración y pacientes, podrás conocer de primera mano la propuesta de SEFAC en este campo.

Esta apuesta decidida por la colaboración interdisciplinar vertebrará la mesa redonda en la que se abordará el asunto de la continuidad asistencial, con el espíritu de sumar las fortalezas de la 
farmacia comunitaria y hospitalaria, o en la dedicada a materia de cronicidad, que recoge el espíritu del documento firmado en noviembre del pasado año por SEFAC y las principales sociedades médicas de atención primaria, como SEMERGEN, semFYC o SEMG, con el objeto de consensuar un modelo de coordinación entre ambos colectivos para atender al paciente crónico. El ánimo de suma al que siempre tienden los congresos SEFAC, donde siempre ha primado estrechar lazos y compartir conocimientos entre profesionales sanitarios, también se dejará sentir en las sesiones compartidas con médicos de primaria y la sesión conjunta con la Agencia Española de Medicamentos y Productos Sanitarios, donde presentaremos actividades conjuntas sobre el uso racional de antibióticos en el marco del Plan Nacional para el uso responsable de estos medicamentos.

En el programa científico de esta octava edición del congreso hemos procurado que se alternen con fluidez evidencia científica y conocimientos prácticos. La primera estará bien presente en sesiones donde se disertará sobre aquellos avances en farmacogenética -que resultarán claves en el desarrollo de fármacos personalizados-, epigenética, avances y retos en cáncer de mama y salud ocular o fundamentos científicos de la fotoprotección sistémica y los nutracéuticos. Y es que conviene decirlo alto y claro: del ámbito de la farmacia pueden surgir grandes investigadores. Uno de los ejemplos más ilustres en este sentido es el reputado catedrático y farmacéutico Juan Carlos Izpisua, que por primera vez participa en un congreso para farmacéuticos comunitarios y que nos hará el inmenso honor de inaugurar el evento, con una fascinante conferencia inaugural sobre la medicina regenerativa en la que se disertará sobre la posibilidad de frenar el envejecimiento.

En Alicante abundarán también sesiones prácticas orientadas a mejorar la práctica asistencial del día a día: cómo determina la genética la efectividad de las terapias, qué puede hacer el farmacéutico para evitar los cólicos de lactante o cómo se pueden abordar desde la farmacia los trastornos del estado de ánimo son sólo algunos de los temas que se tratarán en este octavo congreso de SEFAC, donde además se desmontarán los mitos más arraigados en torno a la anticoncepción de urgencia a través de una serie de casos prácticos, y se reflexionará sobre tendencias de futuro que podrían modificar la misma práctica farmacéutica. Así, una de las mesas redondas introducirá el concepto de farmacogenética, o cómo dotar a la farmacia de información y herramientas para convertir al farmacéutico comunitario en un activo en salud.

Mención aparte merecen los servicios profesionales farmacéuticos, de cuya consolidación y difusión depende el futuro mismo de la profesión. Los asistentes al congreso tendrán la oportunidad de asistir a un buen número de sesiones en las que se instruirá sobre su implantación, y conocerán de primera mano aquellas tecnologías que les pueden permitir implantar dichos servicios en sus farmacias. En Alicante demostraremos el impacto que supone la intervención del farmacéutico comunitario en la cesación tabáquica, con la presentación de los resultados farmacoeconómicos del mayor estudio realizado hasta la fecha en farmacia comunitaria, que avalan la importancia de programas de capacitación como CESAR.

\section{Presencia internacional destacada}

El congreso de Alicante será también el más internacional, consolidando una tendencia que ya se venía repitiendo en las últimas ediciones y que nos ha llevado a institucionalizar la llamada I Reunión Internacional de Farmacéuticos Comunitarios. Personalidades tan relevantes como Christine Bond, Bruno Galan o Ema Paulino compartirán con los asistentes casos de éxitos de la farmacia asistencial europea, y explicarán las claves de los modelos de atención y retribución farmacéutica de países como Escocia, Francia o Portugal. En materia de rigidez arterial contaremos con primeros espadas de Portugal y Argentina, como los doctores Pedro Cunha y Pedro Forcada, que nos contarán los últimos avances al respecto vinculados a un nuevo paradigma en el inicio y seguimiento de la terapia de antihipertensivos, como es la determinación de la rigidez arterial en farmacia comunitaria.

Presencia internacional también encontraremos en la mesas sobre adherencia, en la que expertos internacionales en la materia, como el profesor británico Rob Horne, expondrá a los asistentes las características la experiencia del Reino Unido en esta materia. Junto a él contaremos con expertos de otras profesiones como la médica, que tendrán cabida en otras muchas sesiones (cronicidad, sistemas personalizados de reacondicionamiento...) para reforzar la línea de colaboración entre médicos y farmacéuticos en beneficio de los pacientes que, a su vez, también tendrán su hueco en el programa para expresar su opinión sobre el rol de los farmacéuticos.

El congreso SEFAC 2018 se contagiará de la vitalidad e inconfundible luz de la ciudad que lo alberga. La víspera del congreso se instalará una carpa de servicios profesionales abierta a la población y durante sus tres días de duración los pasillos del Auditorio de Alicante y los lugares más emblemáticos de la ciudad se llenarán de actividades cardiosaludables y asistenciales. Pero además, queremos que tu estancia en Alicante sea inolvidable: hemos diseñado un programa de actividades extracongresuales que te llevarán a descubrir los rincones más emblemáticos de este faro del Mediterráneo, como el Castillo de Santa Bárbara.

Como acredita el reconocimiento de acontecimiento de interés sanitario que nos ha concedido el Ministerio de Sanidad, Servicios Sociales e Igualdad, aspiramos a que Alicante sea un salto adelante para la profesión en la que se plasmen las aspiraciones, realidades y posibilidades de la farmacia asistencial, una cita imprescindible para aquellos farmacéuticos que quieren estar en la vanguardia de la profesión, pero también para todos aquellos agentes sanitarios dispuestos a abrir nuevas vías de colaboración interprofesional en beneficio del paciente. Te esperamos en Alicante para que nos ayudes a tallar el material del que están hechos los sueños profesionales. 